\title{
Manejo microquirúrgico de fístula arteriovenosa dural tentorial entre arteria de Bernasconi-Cassinari y vena de Galeno guiada por angiografía intraoperatoria: reporte de caso y revisión de la literatura Microsurgical management of tentorial dural arteriovenous fistula between artery of Bernasconi-Cassinari and vein of Galen guided by intraoperative angiography: case report and review of literature
}

Andrea Sandoval-Otero MD. ${ }^{1,4}$, David Vergara-García MD. ${ }^{1,2,3,4}$, Jeferson Arango-Rodríguez MD. ${ }^{1,4}$, Alberto Caballero MD. ${ }^{1,4}$, Jorge Torres MD. ${ }^{1,2,4}$

1 Departamento de Neurocirugía, Hospital Universitario de la Samaritana. Bogotá, Colombia.

2 Servicio de Neurocirugía, Hospital Universitario Mayor, Hospital Universitario Barrios Unidos, Méderi. Bogotá, Colombia.

3 Programa de posgrado de Neurocirugía, Universidad del Rosario. Bogotá, Colombia.

4 Centro de Entrenamiento e Investigación en Neurocirugía, Hospital Universitario de la Samaritana. Bogotá, Colombia.

Rev. Chil. Neurocirugía 45: 241-245, 2019

\begin{abstract}
Resumen
Las fístulas arteriovenosas durales (FAVDs) son conexiones vasculares anómalas entre arterias durales y senos venosos o venas corticales encefálicas. Estos cortocircuitos pueden cursar con isquemia o hemorragia intracraneanas que derivan del aumento en el drenaje de los senos venosos o de la hipertensión venosa cortical, implícitos en su fisiopatología. La técnica 'Gold standard' para su diagnóstico es la angiografía cerebral por sustracción digital, sin embargo, existen diferentes técnicas de imagen que aportan información importante para su caracterización y clasificación. Dentro de estos sistemas de clasificación tenemos el sistema Borden-Shucart y la escala de Cognard, que son los más utilizados. Las estrategias terapéuticas para su manejo incluyen métodos endovasculares y/o quirúrgicos. En términos generales el manejo endovascular ha reemplazado al manejo quirúrgico en los últimos años, dado que se asocia a menor tasa de morbimortalidad (mortalidad < $9 \%$ y menor tiempo de estancia hospitalaria. Las fístulas arteriovenosas durales tentoriales (FAVDTs) corresponden al 4 a $8 \%$ del total de FAVDs y son el subtipo con mayor riesgo de sangrado, que se presenta en 38 a $74 \%$ de los pacientes. En el presente artículo se describe el caso de un paciente adulto con una FAVDT entre la arteria de Bernasconi-Cassinari y la vena de Galeno asociada a hemorragia mesencefálica. El paciente fue llevado a manejo microquirúrgico guiado por angiografía intraoperatoria, obteniendo una oclusión completa del defecto fistuloso sin complicaciones y con adecuada evolución postoperatoria.
\end{abstract}

Palabras clave: Tentorial, fístula arteriovenosa dural, endovascular, neurovascular, microcirugía.

\begin{abstract}
Dural arteriovenous fistulas (DAVFs) are anomalous vascular connections between dural arteries and venous sinuses or cerebral cortical veins. These shunts can present with intracranial ischemia or hemorrhage that result from increased venous drainage or cortical venous hypertension, implicit in its pathophysiology. The "gold standard" technique for diagnosis is cerebral digital subtraction angiography, however, there are different imaging techniques that provide important information for its characterization and classification. The Borden-Shucart system and the Cognard scale are the best known classification systems. Therapeutic strategies for its management include endovascular and/or surgical methods. In general terms, in recent years endovascular
\end{abstract}


management has replaced surgical management since it is associated with a lower rate of morbidity and mortality (mortality < $9 \%$ ) and shorter hospital stay. Tentorial dural arteriovenous fistulas (TDAVFs) account for4 to $8 \%$ of all DAVFs and they are the subtype with the highest risk of bleeding, which occurs in 38 to $74 \%$ of patients. In the present article we describe the case of an adult patient with a TDAVF between the Bernasconi-Cassinari artery and the Galen vein associated with mesencephalic hemorrhage. The patient underwent microsurgical management guided by intraoperative angiography, obtaining complete occlusion of the fistulous defect with no complications and with an adequate postoperative evolution.

Key words: Tentorial, dural arteriovenous fistula, endovascular, microsurgery.

\section{Introducción}

Las fístulas arteriovenosas durales (FAVDs) son conexiones vasculares anómalas entre arterias durales y senos venosos o venas corticales encefálicas. Representan aproximadamente un 10 a $15 \%$ de las malformaciones vasculares intracraneales. Tienen un pico de incidencia entre los 50 a 60 años, y no presentan predilección de género ${ }^{1,2,3,4}$. Las fístulas arteriovenosas durales tentoriales (FAVDTs) corresponden a un subtipo de FAVDs que poseen mayor riesgo intrínseco de sangrado, isquemia o déficit neurológico progresivo ${ }^{1,5}$. Las FAVDTs de la arteria tentorial media de Bernasconi-Cassinari, aunque raras, son un ejemplo de éstas.

El tratamiento de este tipo de lesiones puede realizarse a través de técnicas endovasculares o microquirúrgicas, siendo las primeras las de mayor auge en la actualidad por menor tasa de morbimortalidad asociada. Sin embargo, las técnicas microquirúrgicas, que ofrecen mejores tasas de oclusión, permanecen siendo una alternativa viable para aquellos casos donde no sea posible la realización de técnicas endovasculares ${ }^{7,4}$. Se describe el caso de un paciente adulto con una FAVDT de la arteria tentorial media de Bernasconi-Cassinari a la vena de Galeno, quien fue sometido a manejo microquirúrgico guiado por angiografía intraoperatoria.

\section{Reporte de caso}

Paciente de 30 años de edad que acudió al servicio de urgencias del Hospital Universitario de La Samaritana por cuadro clínico de 15 días de evolución consistente en cefalea holocraneana opresiva, de moderada a severa intensidad, asociada a sensación vertiginosa. La sintomatología inició posterior a un trauma craneoencefálico leve, sin otros hallazgos neurológicos asociados. Por esta razón se indico la toma de una resonancia magnética cerebral simple que evidenció presencia de restos hemáticos a nivel del tectum y tegmentum mesencefálico izquierdo (Figuras 12 ), con escaso edema vasogénico asociado y sangrado intraventricular en el asta occipital izquierda.

Ante la sospecha de una FAVD se solicitó una panangiografía cerebral con sustracción digital (Figura 3), la cual demostró la presencia de una FAVDT de la arteria tentorial media de BernasconiCassinari a la vena de Galeno izquierda, Cognard III, Borden III.

El paciente fue llevado a oclusión microquirúrgica (Figura 4) de la fístula a través de un abordaje subtemporal (Figura 5), lo cual permitió la exposición del borde libre del tentorio. Durante el procedimiento quirúrgico se realizó una panangiografía cerebral con sustracción digital (Figura 6) para corroborar la oclusión adecuada del defecto fistuloso luego de clipar la arteria tentorial media de Bernasconi-Cassinari. La inyección de contraste intraarterial postclipaje demostró corrección total del cortocircuito. Durante los dos primeros días del postoperatorio inmediato el paciente permaneció en la Unidad de Cuidados Intensivos para vigilancia neurológica estricta, sin presentar signos de deterioro neurológico. Se realizó una tomografía de cráneo simple de control

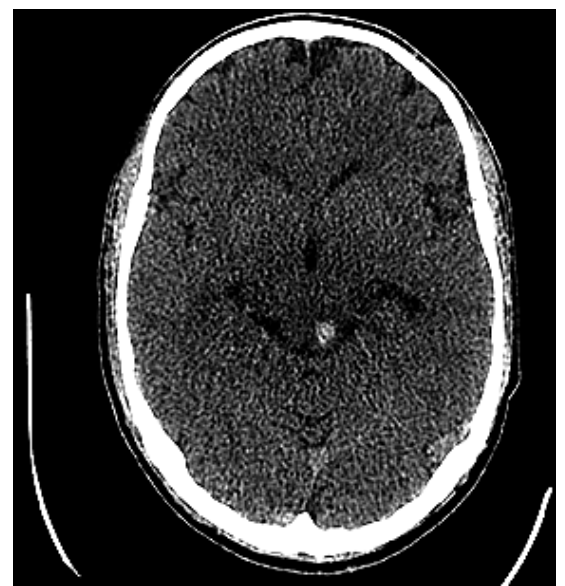

Figura 1. Tomografía de cráneo simple de ingreso con evidencia de hematoma intraparenquimatoso mesencefálico izquierdo.

postoperatoria con adecuados cambios postquirúrgicos y sin evidencia de complicaciones. Dada su buena evolución clínica el paciente fue dado de alta al cuarto día postoperatorio.

\section{Discusión}

Las FAVDs son conexiones vasculares anómalas entre arterias durales y senos venosos o venas corticales encefálicas, que representan un 10 a $15 \%$ de todas las malformaciones vasculares intra-

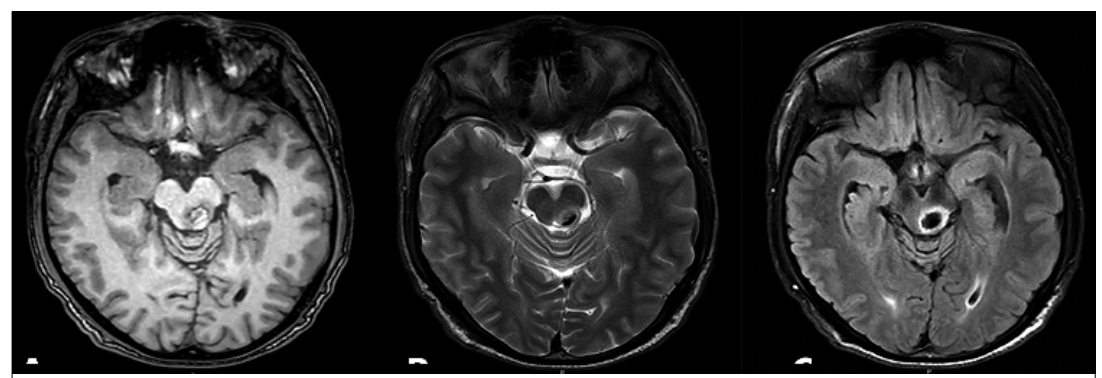

Figura 2. Resonancia magnética cerebral en secuencias $T 1$, T2 y FLAIR con evidencia de restos hemáticos a nivel del tectum y tegmentum mesencefálico izquierdo con escaso edema vasogénico asociado. 


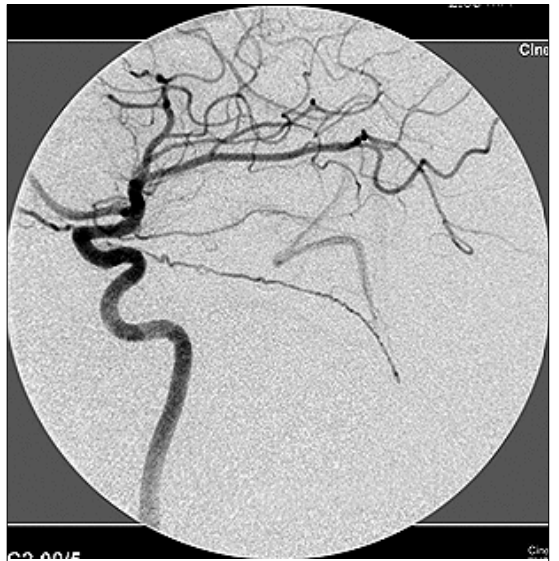

Figura 3. FAVDT de la arteria tentorial media de Bernasconi-Cassinari a la vena de Galeno izquierda, Cognard III Borden-Shucart III.

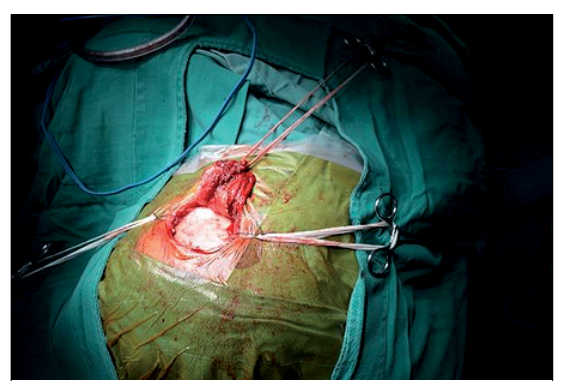

Figura 5. Abordaje subtemporal.

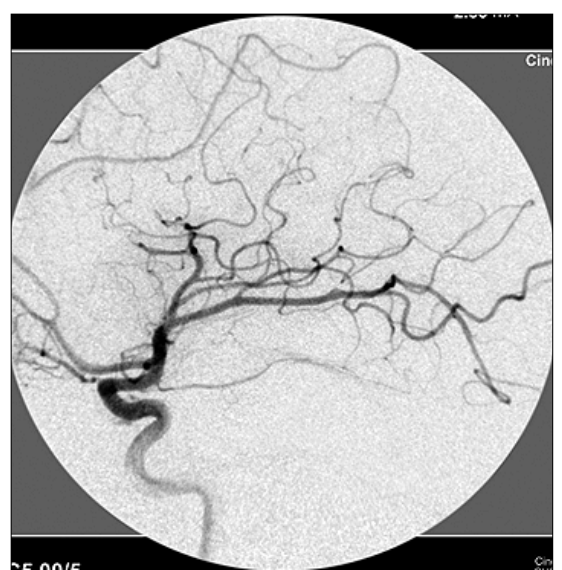

Figura 6. Panangiografía cerebral con sustracción digital intraoperatoria donde se demuestra oclusión completa de la FAVDT.

craneales (6\% de las supratentoriales y $35 \%$ de las infratentoriales). Tienen un pico de prevalencia entre los 50 a 60 años, sin que exista evidencia de predilección de género. Su diagnóstico se ha hecho más frecuente en los últi-

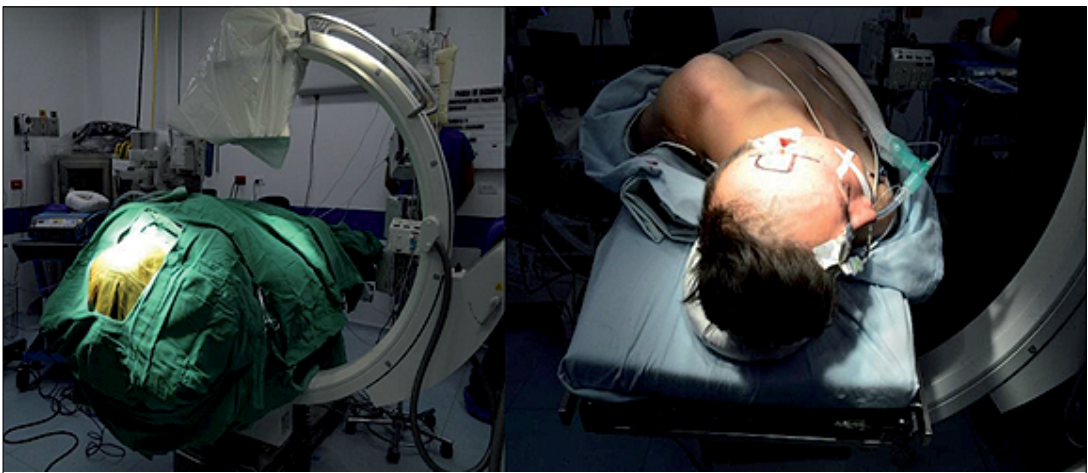

Figura 4. Posicionamiento del paciente en la mesa de cirugía y organización de la sala de para permitir el uso de angiógrafo digital intraoperatorio.

mos años, posiblemente asociado a un aumento en la disponibilidad de equipos de resonancia magnética cerebral ${ }^{1,3,4,6}$. La fisiopatología de estas lesiones usualmente inicia con la trombosis de un seno venoso dural, lo cual conlleva a congestión e hipertensión venosa que genera dilatación de capilares y aparición de fistulas entre el sistema arterial dural y el sistema venoso ${ }^{1}$. Varios estudios han demostrado que la trombosis de senos venosos produce una respuesta inflamatoria con expresión del factor de crecimiento básico fibroblástico (BFGF) y del factor de crecimiento vascular endotelial (VEGF) que, junto con el aumento de la presión venosa, favorecen la creación de cortocircuitos ${ }^{4}$. La mayoría de FAVDs drenan directamente a senos durales venosos, pero con el aumento progresivo de la presión venosa se generan modificaciones histológicas que terminan produciendo proliferación de la íntima vascular venosa que genera reflujo sanguíneo hacia venas corticales. Con el aumento de la presión venosa cortical aparece la predisposición al sangrado intracraneal o a la isquemia ${ }^{1}$. Las manifestaciones clínicas asociadas a las FAVDs se derivan del aumento en el drenaje de los senos venosos o de la hipertensión venosa cortical. Los síntomas asociados con el aumento del volumen de drenaje de los senos venosos dependen de la localización de la lesión (Tabla 1) ${ }^{1}$. Por otro lado, los síntomas asociados con la hipertensión venosa cortical se pueden contemplar como complicaciones hemorrágicas o isquémicas. Las complicaciones hemorrágicas, con una incidencia de 1,5\% anual, son consecuencia de la ruptura de venas corticales frágiles debido al aumento en su presión intraluminal.
La isquemia cortical, por su parte, se produce por congestión venosa que impide el adecuado intercambio de oxígeno con el parénquima encefálico y la depuración de productos del metabolismo ${ }^{1,4,5,6}$. La hipertensión venosa cortical también puede asociarse a la presencia de demencia, parkinsonismo, ataxia, entre otros ${ }^{1,4}$.

Las FAVDs que no cursan con reflujo venoso cortical tienen una historia natural benigna y usualmente corresponden a hallazgos incidentales ${ }^{1}$. De estas sólo el $1 \%$ desarrolla sangrado intraparenquimatoso. Aquellas que sí cursan con reflujo venoso cortical tienen un mayor riesgo de producir déficit neurológico progresivo o sangrado intraparenquimatoso $(35 \%)^{1,5,6}$. Las FAVDTs corresponden al 4 a $8 \%$ del total de FAVDs, y son el subtipo con mayor riesgo de sangrado. Presentan un comportamiento agresivo en el 79 a $92 \%$ de los casos, y producen sangrado en 38 a $74 \%$ de los pacientes ${ }^{3}$. Usualmente estas lesiones se relacionan con ramas arteriales del tronco meningohipofisiario (arteria meníngea dorsal, arteria hipofisiaria inferior y arteria tentorial media de Bernasconi-Cassinari), pero también pueden encontrarse otras arterias involucradas (ej. la arteria meníngea media, la arteria meníngea posterior y las arterias occipitales) ${ }^{2}$.

El estudio 'gold standard' para el diagnóstico de esta patología es la arteriografía cerebral con sustracción digital ó panangiografía cerebral. Otros estudios que pueden proveer datos complementarios son la tomografía computarizada de cráneo simple (TAC), la resonancia magnética (RMN) cerebral simple y la angiografía por TAC o resonancia. La tomografía computarizada simple no es el estudio ideal para identificar la presencia 
Tabla 1.

Síntomas asociados al aumento del volumen de drenaje de los senos venosos según su localización

\begin{tabular}{|c|c|}
\hline Seno & Clínica \\
\hline Seno cavernoso & $\begin{array}{l}\text { - Proptosis } \\
\text { - Quemosis } \\
\text { - Oftalmoplejía } \\
\text { - Disminución de la agudeza visual } \\
\text { - Dolor retro-ocular }\end{array}$ \\
\hline Seno transverso o sigmoide & - Tinitus \\
\hline Seno sagital superior & $\begin{array}{l}\text { - Hipertensión endocraneana } \\
\text { - Papiledema } \\
\text { - Crisis epilépticas } \\
\text { - Demencia }\end{array}$ \\
\hline
\end{tabular}

Tabla 3.

Clasificación Cognard

\begin{tabular}{|l|l|}
\hline Tipo & Características \\
\hline I & Drenaje anterógrado a senos venosos durales \\
\hline Ila & Drenaje retrógrado a senos venosos durales \\
\hline Ilb & Drenaje anterógrado a senos venosos durales y venas corticales \\
\hline Ila+b & Drenaje retrógrado a senos venosos y venas corticales \\
\hline III & Drenaje a venas corticales sin ectasia \\
\hline IV & Drenaje a venas corticales con ectasia \\
\hline V & Drenaje a venas corticales espinales \\
\hline
\end{tabular}

de estas lesiones, pero sí puede aportar información acerca de complicaciones asociadas. La angiografía por TAC o RMN puede demostrar la presencia de dilatación o congestión vascular. Por último, la RMN pudiera demostrar la presencia de áreas que restringen en la secuencia de difusión, en relación con isquemia, y vacíos de señal en el trayecto de venas ${ }^{1,3,4}$.

Los sistemas de clasificación más utilizados para este tipo de cortocircuito son el sistema Borden-Shucart y la escala de Cognard. El sistema Borden-Shucart (Tabla 2) está basado en el sitio de drenaje venoso, pudiendo ser este un seno venoso o una vena cortical. La escala de Cognard (Tabla 3) está fundamentada en la dirección del drenaje a senos venosos (anterógrado o retrógrado), la presencia o ausencia de drenaje a venas corticales y la arquitectura venosa (venas corticales ectásicas o no ectásicas, o vena espinal perimedular). Estudios han demostrado que las fístulas arteriovenosas durales Cognard tipo Ilb a $\mathrm{V}$ son aquellas que cursan con un mayor riesgo de sangrado intracraneal, isquemia y déficit neurológico ${ }^{1,13}$.

El tratamiento de estos pacientes se realiza a través de manejo quirúrgico y/o endovascular, los cuales pueden ejecutarse en monoterapia o combinados. Diversos factores influyen en la elección del tipo de manejo, incluyéndose el patrón de drenaje, la historia natural de la lesión, la severidad de los síntomas, la edad, el estado general del paciente, las características angiográficas intrínsecas de la lesión, su localización y la morbimortalidad asociada al procedimiento (Tabla 4) $)^{4,7}$.

En términos generales el manejo endovascular ha reemplazado al manejo quirúrgico en los últimos años, dado que se asocia a menor tasa de morbimortalidad (mortalidad < $9 \%$ ) y menor tiempo de estancia hospitalaria ${ }^{3,6,7}$. La embolización transarterial de una FAVD requiere cateterización selectiva del modo que el material de embolización sea llevado al aspecto más proximal de la vena involucrada ${ }^{3,4}$. Diferentes materiales embólicos pudieran usarse, tales como pegamento (NBCA), partículas aspecto distal de la arteria nutricia, de
Tabla 2.

Clasificación Borden-Shucar

\begin{tabular}{|l|l|}
\hline Tipo & Características \\
\hline I & $\begin{array}{l}\text { Drenaje a senos venosos du- } \\
\text { rales o venas meníngeas }\end{array}$ \\
\hline II & $\begin{array}{l}\text { Drenaje a senos venosos y } \\
\text { venas corticales }\end{array}$ \\
\hline III & Drenaje a venas corticales \\
\hline
\end{tabular}

Tabla 4

\begin{tabular}{l}
\hline Patrón de drenaje \\
\hline Historia natural de la lesión \\
\hline Severidad de los síntomas \\
\hline Edad \\
\hline Estado general \\
\hline Características angiográficas \\
\hline Localización \\
\hline Morbi-mortalidad del procedimiento \\
\hline
\end{tabular}

(PVA), Onyx o coils, siendo el Onyx el agente con mayor tasa de oclusión $(62,5 \%)$ comparado con el PVA y el $\mathrm{NBCA}^{4}$. Aun así su efectividad tiende a ser baja, puesto que las arterias involucradas en este tipo de fístulas son de diámetro muy pequeño, lo cual dificulta su completa embolización. Así mismo, este tratamiento no provee disminución en la incidencia de sangrados ni disminución de los efectos secundarios de la hipertensión venosa. Otra de las técnicas endovasculares empleadas es la oclusión transvenosa, que requiere un blanco terapéutico preciso y por ende solo puede considerarse cuando el drenaje de la fistula está separado del drenaje del cerebro normal. Algunos autores no recomiendan esta estrategia terapéutica ya que se relaciona con un riesgo alto de perforación venosa teniendo en cuenta la fragilidad de las paredes de estos vasos ${ }^{3,4}$.

El manejo microquirúrgico es útil cuando el manejo endovascular ha fallado o no es posible. Dentro de las alternativas microquirúrgicas se encuentra el clipaje o la coagulación de las venas leptomeníngeas, la esqueletonización del seno, la oclusión del drenaje venoso, y la coagulación de la fístula y la duramadre adyacente, entre otras ${ }^{2,3}$. Hay reportes de pacientes que alcanzan una tasa de obliteración del $87,5 \%$ si se manejan quirúrgicamente ${ }^{3}$. El uso de un angiógrafo 


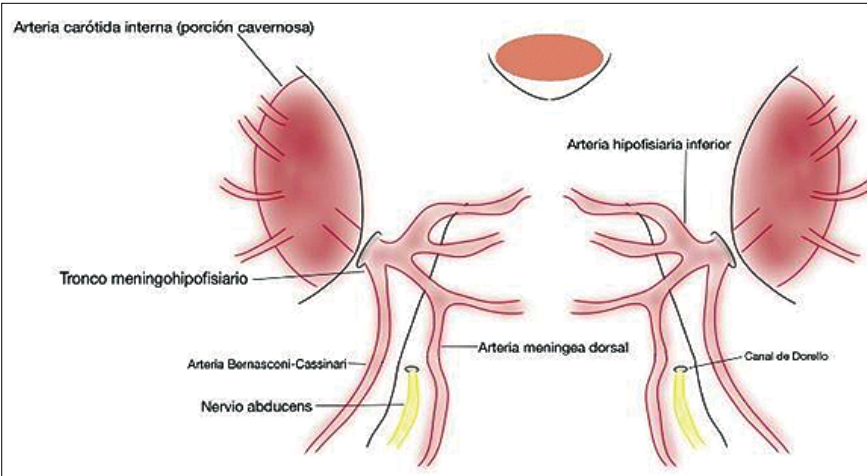

Figura 7. Tronco meningohipofisiario y sus ramas.

intraoperatorio es una herramienta útil para el control preciso de la oclusión selectiva de la(s) arteria(s) nutricia(s) de la FAVD?.

El caso reportado en el presente artículo corresponde a un paciente con una FAVDT con origen nutricio en la arteria tentorial media de Bernasconi-Cassinari (Figura 7) y drenaje a la vena de Galeno (vena cerebral magna), quien debutó con sangrado intraparenquimatoso mesencefálico. Dada la configuración anatómica y ubicación de la fístula (que no permitía una cateterización supraselectiva segura de la arteria nutricia) se consideró realizar manejo microquirúrgico con clipaje la arteria tentorial media de Bernasconi-Cassinari. Durante el procedimiento quirúrgico se hizo uso de panangiografía cerebral con sustracción digital para asegurar cierre total del cortocircuito, obteniendo un excelente resultado postoperatorio.

\section{Conclusiones}

Las FAVDTs corresponden al subtipo de malformaciones arteriovenosas durales con mayor riesgo de complicaciones hemorrágicas. Las intervenciones endovasculares son consideradas la primera línea de tratamiento teniendo en cuenta su baja tasa de morbimortalidad; sin embargo, en casos donde su realización no sea posible o no haya sido exitosa, se debe considerar manejo quirúrgico. La panangiografía cerebral intraoperatoria es una herramienta útil dado que facilita la oclusión selectiva de la(s) arteria(s) nutricia(s) que origina(n) el cortocircuito.

Recibido: 11 de marzo de 2019

Aceptado: 05 de mayo de 2019

\section{Referencias}

1. Chaichana KL, Coon AL, Tamargo RJ, Huang J. (2012). Dural Arteriovenous Fistulas: Epidemiology and Clinical Presentation. Neurosurgery Clinics of North America, 23(1), 7-13. doi:10.1016/j.nec.2011.09.001

2. Liu S, Lee DC, Tanoura T. (2016). Tentorial dural arteriovenous fistula of the medial tentorial artery. Radiology Case Reports,11(3), 242244. doi:10.1016/j.radcr.2016.06.006

3. Zhou L, Chen L, Song D, Gu Y, Leng B. (2007). Tentorial dural arteriovenous fistulas. Surgical Neurology, 67(5), 472-481. doi:10.1016/j. surneu.2006.08.078

4. Santillan A, Nanaszko M, Burkhardt J, Patsalides A, Gobin YP, Riina HA. (2013). Endovascular management of intracranial dural arteriovenous fistulas: A review. Clinical Neurology and Neurosurgery, 115(3), 241-251. doi:10.1016/j.clineuro.2012.11.021

5. Soderman M, Pavic L, Edner G, Holmin S, Andersson T. (2008). Natural History of Dural Arteriovenous Shunts. Stroke, 39(6), 1735-1739. doi:10.1161/strokeaha.107.506485

6. Kim MS, Han DH, Kwon O, Oh C, Han M H. (2002). Clinical characteristics of dural arteriovenous fistula. Journal of Clinical Neuroscience, 9(2), 147-155. doi:10.1054/jocn.2001.1029

7. Gross BA, Ducruet AF, Jankowitz BT, Gardner PA. (2017). An Intraoperative Look at a Residual/Recurrent Tentorial Dural Arteriovenous Fistula. World Neurosurgery,105. doi:10.1016/j.wneu.2017.07.015

8. Tubbs RS, Nguyen HS, Shoja MM, Benninger B, Loukas M, Cohen-Gadol AA. (2011). The medial tentorial artery of Bernasconi-Cassinari: A comprehensive review of its anatomy and neurosurgical importance. Acta Neurochirurgica, 153(12), 2485-2490. doi:10.1007/s00701011-1195-y

9. Banerjee A, Ezer H, Nanda A. (2011). The Artery of Bernasconi and Cassinari: A Morphometric Study for Superselective Catheterization. American Journal of Neuroradiology, 32(9), 1751-1755. doi:10.3174/ajnr.a2552

10. González Ramos J, Ceciliano A, Zuccaro G. (2006). Malformación de la vena de Galeno. Revista Argentina De Neurocirugía.

11. Urueña SM, Malfaz FC, Armengod CG, Pérez AB, Higueras AP. (2003). Malformación arteriovenosa de la vena de Galeno. Anales De Pediatría, 58(6), 580-583. doi:10.1157/13048087

12. Egemen E, Solaroglu I. (2017). Anatomy of Cerebral Veins and Dural Sinuses. Primer on Cerebrovascular Diseases, 32-36. doi:10.1016/ b978-0-12-803058-5.00005-9

13. Cognard C, Gobin YP, Pierot L, Bailly AL, Houdart E, Casasco A, Merland JJ. (1995). Cerebral dural arteriovenous fistulas: Clinical and angiographic correlation with a revised classification of venous drainage. Radiology, 194(3), 671-680. doi:10.1148/radiology.194.3.7862961

\section{Correspondencia a:}

Andrea Sandoval-Otero MD.

Departamento de Neurocirugía, Hospital Universitario de La Samaritana,Bogotá DC, Colombia.

andreasaot@me.com

David Vergara-García MD.

Departamento de Neurocirugía. Hospital Universitario de la Samaritana.

Carrera 8 No. 0-29 Sur, Bogotá DC.

david.vergara@urosario.edu.co 